\title{
On the Existence of Perfectly Isotropic Radiators
}

This paper was downloaded from TechRxiv (https://www.techrxiv.org).

\section{LICENSE}

CC BY 4.0

SUBMISSION DATE / POSTED DATE

$12-01-2022$ / 18-01-2022

\section{CITATION}

Mikki, Said (2022): On the Existence of Perfectly Isotropic Radiators. TechRxiv. Preprint. https://doi.org/10.36227/techrxiv.18263387.v1

$\mathrm{DOI}$

10.36227/techrxiv.18263387.v1 


\title{
On the Existence of Perfectly Isotropic Radiators
}

\author{
Said Mikki
}

\begin{abstract}
We revisit the problem of realizing perfectly isotropic antennas based on recent developments in nonlocal antenna theory. The key obstacle against the existence of exactly isotropic antennas is traced back to the hairy ball theorem in algebraic and differential topology. A path blocking the applicability of this no-go theorem is to allow for complementary longitudinal modes to coexist with matching transverse modes in the antenna exterior region. Nonlocal antennas (radiators embedded into nonlocal metamaterial exterior domains) are possible future antenna technologies capable of dealing with both transverse and longitudinal modes. It is rigorously demonstrated that the recently proposed nonlocal Proca metamaterial (massive electromagnetism) leads to small nonlocal dipole antennas with perfectly isotropic radiation pattern. A tentative proposal for a possible experimental realization of perfectly isotropic radiators in the future is briefly outlined.
\end{abstract}

Index Terms-Antennas, perfectly isotropic radiation, directivity, Proca metamaterial, massive electromagnetism.

\section{INTRODUCTION}

Fundamentally speaking, all antennas radiating in free space or in a temporally-dispersive media (both must be spatially 3-dimensional) can never realize perfectly isotropic radiation patterns. The reason is that in such media the far-field is known to be transverse, i.e., the asymptotic limit of the longitudinal component is exactly zero [1]-[3], while the hairy ball theorem asserts that a continuous vector field on the 2-sphere cannot have a non-vanishing 3-dimensional pattern [4]. ${ }^{2}$ However, such no-go theorem does not preclude attempts to construct practical approximations of isotropic radiators. Indeed, due to the recent rise of attractive and promising technologies such as Radio-frequency identification (RFID), Internet of Things (IoT) and Ubiquitous Computing [5], coupled with the already old trend in electromagnetic applications to push toward antenna miniaturization [6], [7], the general inclination of applied research now favors implanting or embedding small antennas into many (if not all) key objects found in our surrounding environments. However, since everyday objects are randomly oriented in 3-dimensional space, while the transmit/receive properties of antennas can be very sensitive to polarization data [8], it is essential that these antennas posses a perfectly isotropic radiation (PIR) pattern in order to ensure reliability and consistency in the overall system performance. Indeed, signals received or transmitted along nulls in the radiation pattern readily lead to jammed reception or fading in the corresponding wireless communication link [9].

The principal objective of this article is to reexamine perfectly isotropic radiators at a general and broad fundamental level by shedding new insights into the subject, especially in

\footnotetext{
${ }^{1}$ The author is with Zhejiang University/University of Illinois at UrbanaCampaign (ZJU-UIUC) Institute, Haining, Zhejiang, China. He can be reached at said.m.mikki@gmail.com

${ }^{2} \mathrm{Cf}$. Sec. II for complete details.
}

light of recent related research developments. While a rather large literature exist on how to design practical devices with partially isotropic radiation patterns, involving most kinds of important antennas, conducted both numerically and experimentally, ${ }^{3}$ it is the opinion of this author that the subject of why and how antennas can (or cannot) have perfectly isotropic radiation pattern has not received a sufficient attenuation from the fundamental viewpoint. In what we follows, we approach this old subject with a fresh perspective stimulated by the now emerging area of nonlocal metamaterials [15]-[19], especially the recently proposed research field in antenna theory called nonlocal antenna theory [20], [21].

This paper is structured as follows. In Sec. II, we start by rigorously formulating the problem of the existence of PIR pattern for local antennas ${ }^{4}$ in terms of a no-go theorem, the hairy ball theorem. The main conditions blocking the existence of PIR patterns in this class of radiators are formally identified and then exploited in Sec. III by introducing the concept of the Proca antenna, a special example of a nonlocal antenna discovered recently [23]. It is carefully shown that for this class of problems a theorem asserting the existence of PIR patterns can be proved by using the concept of complementary radiation patterns introduced in Sec. II. Next, we provide in Sec. IV a tentative proposal for a possible future realization of PIR antennas through an approximation of the ideal Proca antenna described in Sec. III. Finally, we end up with conclusions and recommendations for how to extend this work.

\section{The Hairy Ball Theorem as a No-Go Result on Perfectly Isotropic Antennas in Local Domains}

To simplify our treatment, we work in the frequency domain, i.e., we assume that all our electromagnetic fields are excited by a sinusoidal source $\exp (-\mathrm{i} \omega t)$ with frequency $\omega$. The fields are then harmonic complex vector field (but with the time-dependence removed) denoted by $\mathbf{E}(\mathbf{x}), \mathbf{H}(\mathbf{x}) \in \mathbb{R}^{3}$, where $\mathbf{x} \in \mathbb{R}^{3}$ is the position vector and we suppress the explicit dependence on $\omega$ whenever possible but all fields, and consequently radiation patterns, remain frequency dependent. The question of how to formulate a perfectly isotropic radiator is then equivalent to asking whether the far-zone fields, defined by the asymptotic limits [3], [24],

$$
\mathbf{E}^{\mathrm{far}}(\Omega):=\|\mathbf{x}\| \lim _{\mathbf{x} \rightarrow \infty} \mathbf{E}(\mathbf{x}), \mathbf{H}^{\mathrm{far}}(\Omega):=\|\mathbf{x}\| \lim _{\mathbf{x} \rightarrow \infty} \mathbf{H}(\mathbf{x}),
$$

have no variations in the angular directions $\Omega:=(\theta, \varphi)$, where $\theta$ and $\varphi$ are the spherical coordinates angles. More precisely,

\footnotetext{
${ }^{3}$ We will not survey this literature since our objective is not to provide a review of isotropic antennas. However, see for example [10]-[14].

${ }^{4}$ Nonlocal antenna systems are defined as sources radiating into free space or temporally dispersive media [20], [22].
} 
we define the perfectly isotropic radiator (PIR) system as follows:

Definition 1. (Perfectly isotropic radiators) For a given set of far fields $\mathbf{E}^{\mathrm{far}}(\Omega)$ and $\mathbf{H}^{\mathrm{far}}(\Omega)$, we say that the radiating system possesses a perfectly isotropic radiation (PIR) pattern when the two conditions

$$
\nabla_{\Omega} \mathbf{E}^{\mathrm{far}}(\Omega ; \omega)=\nabla_{\Omega} \mathbf{H}^{\mathrm{far}}(\Omega ; \omega)=0
$$

are satisfied simultaneously for every $\omega>0$. If only the electric (magnetic) field condition is satisfied, we say we have an electric (magnetic) type perfectly isotropic radiating system.

Next, we present the main result from the theory of continuous manifolds prohibiting the existence of PIR systems in local domains. In both algebraic and differential topology, the following theorem is fundamental [4]

Theorem 1. (The hairy ball theorem) Consider a continuous vector field $g: \mathbb{R}^{n} \rightarrow \mathbb{R}^{n+1}$, where $n$ is a positive integer. Let $N(x) \in \mathbb{R}^{n+1}$ be the vector normal to the $n$-sphere $\mathcal{S}_{n}$ at the point $x \in \mathcal{S}_{n}$. Assume that the following two conditions are satisfied:

1) $n$ is even.

2) $g(x) \cdot N(x)=0$ for all $x \in \mathcal{S}_{n}$, where $a \cdot b$ is the Euclidean inner product for vectors in the ambient space $\mathbb{R}^{n+1}$.

Then $g(x)$ must vanish at least at one point $x \in \mathcal{S}_{n}$.

For the problem of electromagnetic radiation, the configuration space is the position space $\mathbf{x} \in \mathbb{R}^{3}$ where the frequency $\omega$ is fixed. The relevant far-field zone is the 2sphere $\mathcal{S}_{2}$ parameterized by $\Omega$ (a 2-dimensional manifold [4]..$^{5}$ ) Consequently, $n$ in Theorem 1 is 2, i.e., it is even (first condition is met).

Now, regarding the second condition, the nature of the medium surrounding the antenna plays a fundamental role. If the antenna's exterior region $D_{\text {ext }}$ is filled with either free space or a temporally dispersive medium, ${ }^{6}$ then it can be shown based on general principles that the far-fields are tangential to the infinite sphere $\mathcal{S}_{2}$, i.e., we have in this case:

$$
\mathbf{E}^{\mathrm{far}}(\Omega) \cdot \hat{r}=\mathbf{H}^{\mathrm{far}}(\Omega) \cdot \hat{r}=0 .
$$

Here, $\hat{r}$ is the spherical coordinates radial vector plays the role of $N(x)$ in Theorem $1 .^{7}$ Therefore, the two conditions of the theorem are met and we may conclude the following:

Theorem 2. (Nonexistence of perfectly isotropic local antennas) Consider an arbitrary local antenna, i.e., a current source distribution $\mathbf{J}(\mathbf{x})$ such that it radiates continuous fields

\footnotetext{
${ }^{5}$ Strictly speaking, a sphere cannot be fully parameterized by the spherical angles $\theta$ and $\varphi$ but one must use a system of overlapping coordinates [4] However, this subtlety has no bearing on what follows so whenever needed a coordination of the sphere by $\Omega$ is to be understood as a system of complete differential atlas.

${ }^{6} \mathrm{~A}$ temporally dispersive medium is a material domain with constitutive parameters (scalar or tensors) that in the frequency domain depends only on $\omega$. If the medium's response parameters also depend on $\mathbf{k}$, the wavevector, then the medium becomes nonlocal (or spatially dispersive if the material is also homogeneous) [18], [19], [25].

${ }^{7}$ The relation (3) can be easily proved from Wilcox expansion, e.g., see [3], [24], [26], [27].
}

into an exterior domain $D_{\text {ext }}$ where the latter is either free space or a material domain filled with a local medium (i.e., a temporally dispersive material). Then there exists no current source $\mathbf{J}(\mathbf{x})$ such that the antenna can give rise to a perfectly isotropic radiator in the sense of Definition 1.

Theorem 2 provides a rigorous modern mathematical statement of the well known fact about the practical impossibility of constructing an actual perfectly isotropic radiators in free space. It also extends this fundamental impossibility to generic (isotropic or anisotropic) media with arbitrary dependence of their material tensors on $\omega$. Indeed, in all such antenna systems the far field cannot possess a radial component and hence the hairy ball theorem requires the existence of at least one null in the radiation pattern. Since the far-field pattern is continuous, it follows that the radiation characteristics of the system, whether in the transmission or (using reciprocity) receive mode, must experience rapid degradation in at least a small angular sector around the null's location.

\section{Proca Antennas, Nonlocality, And the EXISTENCE OF PERFECTLY ISOTROPIC RADIATORS}

A path to escape from the No-Go Theorem 2 is provided by the fact that the classical asymptotic Wilcox expansion [27] is no longer valid in generic nonlocal or spatially dispersive media [24], [25], [28], [29]. New or "additional waves" must be incorporated in the solution of Maxwell's equations in order to remain consistent with nonlocality [30], [31]. To the best of the author's knowledge, a complete mathematical treatment of the general structure of radiation fields in generic nonlocal media currently does not exist. However, this problem was reconsidered recently in homogeneous nonlocal media, i.e., the important case of spatial dispersion. The general structure of the radiation field was derived in [20] using a momentum (Fourier) space method [22]. In such an approach, all fields are expanded in temporal and spatial Fourier frequencies $\omega$ and $\mathbf{k}$, respectively. When applications and examples of various nonlocal antennas were developed using this general theory, it was initially noted that if optimization methods are applied to nonlocal antenna systems, then it might lead to perfectly isotopic radiators [21].

The logic behind this conjecture is briefly the following. In a generic nonlocal metamaterial (MTM), both transverse (T) and longitudinal (L) waves can be excited [32]. However, similar to what happens in plasma waves [28], longitudinal modes in a generic nonlocal domain may carry energy to the far zone [20], [29]. Now, following the construction of the radiation pattern using the energy method in [20], [22], one may look for special combinations of $\mathrm{L}$ and $\mathrm{T}$ modes such that the total radiation pattern is perfectly symmetric. The hope is that an optimization of a series of properly excited L and $\mathrm{T}$ modes may lead to the formation of exact or nearly-exact complementary radiation patterns. We propose the following definition:

Definition 2. (Complementary transverse-longitudinal field system) Let $\mathbf{E}^{\mathrm{T}}(\Omega)$ and $\mathbf{E}^{\mathrm{L}}(\Omega)$ denote the total radiation patterns due to all the transverse (T) and longitudinal (L) 
modes, respectively. We say that the pair $\left(\mathbf{E}^{\mathrm{T}}(\Omega), \mathbf{E}^{\mathrm{L}}(\Omega)\right)$ constitutes a T-L complementary field system if the condition

$$
\nabla_{\Omega}\left[\mathbf{E}^{\mathrm{T}}(\Omega ; \omega)+\mathbf{E}^{\mathrm{L}}(\Omega ; \omega)\right]=0
$$

is satisfied at all angles $\Omega \in 4 \pi$ and every frequency $\omega>0$. In other words, while each of $\mathbf{E}^{\mathrm{T}}(\Omega)$ and $\mathbf{E}^{\mathrm{L}}(\Omega)$ can be a strong function of $\Omega$, the two fields' respective angular dependencies exactly cancel when added to each other.

Note that the mere definition of the commentary field system in Definition 2 does not guarantee the existence of interesting physical examples. After all, the fields radiated by a physically realizable antenna system must satisfy Maxwell's equations and the material constitutive relations. Remarkably, it was discovered recently that a complementary field system may exist in Proca theory, i.e., the theory of massive electromagnetism [23]. In such theory, the electromagnetic fields possess a non-Gauge invariant structure where photons have a nonvanishing mass [33], [34]. However, it was shown that a special type of nonlocal MTMs, hereafter Proca MTM, can lead to exact equivalence between Maxwell's and Proca's field theories, where the electromagnetic fields exactly solves Proca's equation (massive electromagnetism) in vacuum [23]. In other words, massive Proca fields in vacuum are formally equivalent to Maxwell's theory in a nonlocal (spatially dispersive) medium, the Proca MTM. Most noteworthy is the fact that the fields radiated by an infinitesimal (point) source (Proca source) are perfectly isotropic regardless to the orientation of the infinitesimal dipole:

Theorem 3. (Existence of perfectly isotropic nonlocal antennas) Let $\mathbf{J}(\mathbf{r}, t)=\hat{\alpha} J \delta\left(\mathbf{r}-\mathbf{r}_{s}\right) \exp (-\mathrm{i} \omega t)$ be the current of a time harmonic infinitesimal dipole oriented along $\hat{\alpha}$ and located at $\mathbf{r}_{s}$, while resonating at frequency $\omega$. Let $P(\omega, \hat{\alpha} ; \Omega)$ be the corresponding momentum space radiation power density. ${ }^{8}$ Then if the source is embedded into a Proca MTM, it follows that

$$
\nabla_{\hat{\alpha}} \nabla_{\Omega} P(\omega, \hat{\alpha} ; \Omega)=0
$$

for all frequencies $\omega>0$ angular directions and dipole orientations $\Omega, \hat{\alpha} \in 4 \pi$.

Proof sketch. This is a direct consequence of Definition 2 and Theorem 3, Appendix A.4, [23] after utilizing (A.16) and (A.17) in [23] in order to compute the radiation fields. Note that in (5) we treat $\hat{\alpha}$ and $\Omega$ as independent direction variables. ${ }^{9}$

\section{ON THE Realization OF PeRfectly Isotropic Radiators Using Proca Antennas}

The goal of the present section is provide a provisional proposal on how to use Theorem 3 to outline a possible

\footnotetext{
${ }^{8}$ See [20], [22] for detailed definition of the momentum space approach to radiation energy and power densities in general. For various examples, see [21], [23].

${ }^{9}$ The expression (5) indicates that the perfectly isotropic nature of the Proca source is not an accident of orienting the antenna along a special direction but is valid for any dipole orientation (as it should be in a truly perfectly symmetric radiation configuration)
}

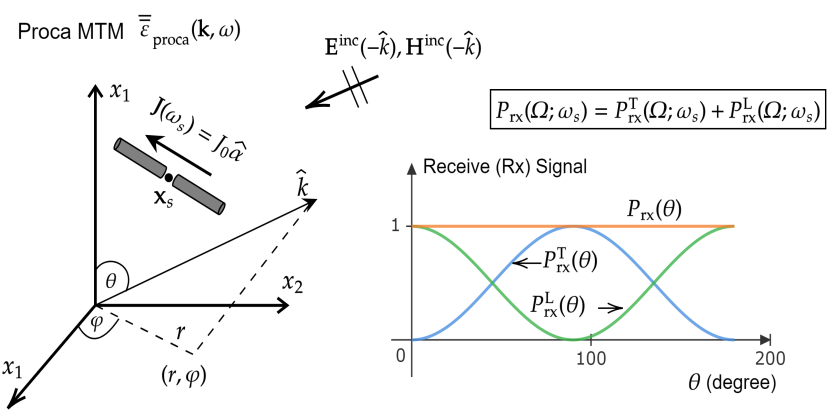

Fig. 1: The general configuration of the proposed perfectly isotropic Proca antenna system operating at frequency $\omega_{s}$ and oriented along $\hat{\alpha}$ while embedded into an infinite and homogeneous Proca MTM (6).

path toward future research on new generations of advanced nonlocal antennas with isotropic radiation pattern.

Let us first review the generalized (effective) dielectric tensor of the Proca MTM in such formulation, which was found to expressed in the following surprisingly simple analytical form [23]:

$$
\overline{\bar{\varepsilon}}_{\text {proca }}(\mathbf{k}, \omega)=\left(1-\frac{m^{2} c^{2}}{\omega^{2}}\right) \overline{\mathbf{I}}-\frac{k^{2} c^{2}}{\omega^{2}} \hat{k} \hat{k} .
$$

Here, $\overline{\mathbf{I}}$ is the unit dyad. The normalized mass $m$ is the fundamental parameter of Proca theory, which is given in terms of the photon mass $m_{\mathrm{ph}}$ through the relation $m=m_{\mathrm{ph}} c / \hbar$, where $\hbar$ is the reduced Plank constant and $c$ the speed of light [33]-[35]. The quantity $m$ possesses inverse length units (in SI we use $\mathrm{m}^{-1}$ ), and hence $1 / \mathrm{m}$ is a characteristic length scale, which turned out to be related to the nonlocality radius of the Proca of MTM [23]. Extensive in-depth discussion of the physical meaning of the quantities $m$ and $1 / m$ can be found in [23]. For our immediate purposes here, we mention that the Proca MTM's material tensor (6) may in principle be realized in the lab provided a method to synthesize both the $\mathrm{T}$ and $\mathrm{L}$ response tensors (the components proportional to $\overline{\mathbf{I}}$ and $\hat{k} \hat{k}$, respectively) can be devised. ${ }^{10}$ Only Proca waves can be excited in a Proca MTM. These are a combination of $\mathrm{T}$ and $\mathrm{L}$ waves with identical dispersion relations given by $\omega^{2}=c^{2} k^{2}+c^{2} m^{2}$. It should be noted that for a Proca antenna to effectively radiate into the Proca MTM, i.e., the Proca's source exterior domain, the operating frequency must exceed the cutoff frequency of the Proca system (see [23] for details.)

Fig. 1(left) illustrates a basic example of an exact Proca source, i.e., an infinitesimal dipole embedded into an infinite and homogeneous nonlocal Proca MTM with a dielectric tensor as in (6). We used the transmitting mode characteristic of the ideal Proca source, computed by (61) and (62) in [23], after which the Lorentz reciprocity theorem [36] was invoked in order to relate the receive power pattern signal to the transmitting antenna radiation pattern. The reciprocity theorem [37] is applicable because the Proca MTM is both passive

\footnotetext{
${ }^{10}$ Several Proca MTM design algorithms were proposed in [23]; inherent related design tradeoffs were discussed there too.
} 
and linear [23]. The Proca antenna is placed in the receive mode in order to test its perfectly isotropic pattern, where an incident plane wave system $\left(\mathbf{E}^{\text {inc }}, \mathbf{H}^{\text {inc }}\right)$ is applied. Using the momentum space formalism, the receive power pattern $P_{\mathrm{rx}}(\Omega)$ can be computed directly in terms of the $\mathrm{T}$ and $\mathrm{L}$ mode patterns $P_{\mathrm{rx}}^{\mathrm{T}}(\Omega)$ and $P_{\mathrm{rx}}^{\mathrm{L}}(\Omega)$, respectively, as shown in the top right side of Fig. 1. Here, the plane wave is applied along the direction $-\hat{k}$, while $\hat{k}:=\mathbf{k} / k$ is the momentum-space spectral variable [20]. This allows using the same expressions derived previously using the momentum space formula of the radiation pattern [22] to the problem of receive mode characterization of isotropic dipoles.

The realization of a perfectly isotropic antennas as proposed in Fig. 1, however, faces several challenges. First, one needs to actually construct the Proca MTM itself. While design tradeoffs and algorithms were presented in [23], building a physical layout requires solving several technological problems. Some of them include the ability to control both the $\mathrm{L}$ and $\mathrm{T}$ mode constitutive relations simultaneously; overcoming the trade-off between the size of nonlocality (spatial dispersion strength) in the $\mathrm{L}$ mode dielectric tensor and bandwidth already noted in [23]; and, most importantly, ensuring that the MTM works over a large bandwidth with manageable losses. These issues are outside the scope of the present article but we hope that some or all of these problems will be overcome by future experimental research.

A more essential problem in terms of physical realization might be discussed now assuming a good model of the Proca MTM is at hand. Fig. 2(a) shows a practical realization of the receive system of Fig. 1 effectuated via the use of a finite perfectly symmetrical spherical Proca MTM. Note that in theory Proca MTM, as given by (6), is infinite and homogeneous. On the other hand, in lab realizations the Proca system must be truncated. The finite spherical realization, where an approximation of (6) is placed within a sphere of radius $a$ (Fig. 2a) destroys the perfect symmetry of the problem for the following reason. When an incident place wave interacts with the enclosing surface $S_{\text {proca }}$ of the Proca sphere shown in Fig. 2(a), new waves will emerge into the nonlocal domain, i.e., $\left(\mathbf{E}^{\text {tr }}, \mathbf{H}^{\text {tr }}\right)$, while other waves $\left(\mathbf{E}^{\text {ref }}, \mathbf{H}^{\text {ref }}\right)$ will be reflected. We are more interested now in the transmitted field system $\left(\mathbf{E}^{\operatorname{tr}}, \mathbf{H}^{\text {tr }}\right)$.

It is will known from general considerations that reflection and transmission of waves at material interfaces where one medium is nonlocal (spatially dispersive) leads to the production of new "additional waves" that cannot be predicted by the usual paradigm of boundary-value problems in mathematical physics, the latter has been successfully applied so far mainly to local electromagnetics [19], [25], [28], [29], [38]. The problem of additional waves, whose discovery is essentially due to Pekar [30], [31], makes the computational or even the theoretical analysis of the problem in Fig. 2(a) extremely difficult. The easiest approach might be to actually perform measurement on a realization of a Proca MTM.

Meanwhile, the exact Theorem 3 can not be applied when strong transmitted (spurious) modes such $\left(\mathbf{E}^{\mathrm{tr}}, \mathbf{H}^{\mathrm{tr}}\right)$ reach the dipole at the center as shown in Fig. 2(a). To solve this problem, we propose using a modified inhomogeneous locally-

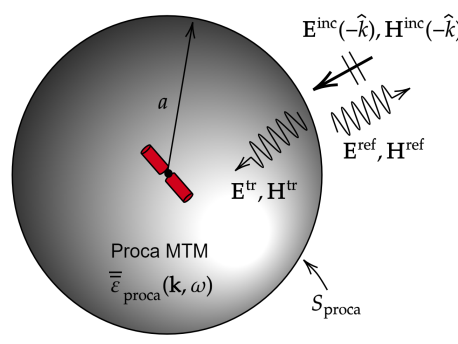

(a)

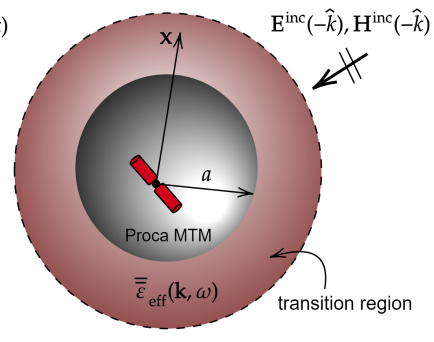

(b)
Fig. 2: (a) Finite spherical realization of an idealized Proca system with sharply discontinuous interface at $S_{\text {proca }}$. (b) A graded transition region is carefully introduced to minimize spurious modes.

spatially-dispersive Proca MTM, ${ }^{11}$ whose dielectric tensor is given by

$$
\overline{\bar{\varepsilon}}_{\text {eff }}(\mathbf{k}, \omega ; \mathbf{x})=\overline{\bar{\varepsilon}}_{\text {proca }}(\mathbf{k}, \omega) \cdot \overline{\mathbf{f}}(|\mathbf{x}| ; a) .
$$

Here, the tensor $\overline{\mathbf{f}}(r, a)$ satisfies the following conditions:

$$
\begin{aligned}
& \text { (i) } \lim _{|\mathbf{x}| \rightarrow \infty} \overline{\mathbf{f}}(|\mathbf{x}| ; a):=\overline{\bar{\varepsilon}}_{\text {proca }}^{-1}(\mathbf{k}, \omega) \varepsilon_{0}, \\
& \text { (ii) } \forall|\mathbf{x}|<a, \overline{\mathbf{f}}(|\mathbf{x}| ; a)=\overline{\mathbf{I}}, \\
& \text { (iii) } \overline{\mathbf{f}}(|\mathbf{x}| ; a) \text { is continuous. }
\end{aligned}
$$

Collectively, these conditions guarantee that for $r<a$, the medium behaves like an ideal Proca MTM (condition (ii)). On the other hand, far away from the dipole, the medium gradually decays or changes into free space (condition (i)). Condition (iii) ensures that the transition does not introduce discontinuities that may lead to the generation of new spurious modes. Collectively, and after a proper optimization, the nonlocal homogeneous model proposed in (7) and (8) should introduce a transition regions (Fig. 2(b)) that acts like a "matching layer" minimizing the production of spurious modes into the test dipole at the center.

\section{CONClusion}

We have formulated the theoretical problem of the existence of perfectly isotropic radiation (PIR) antennas by differentiating the two fundamentally distinct classes of local and nonlocal antennas. After it was illustrated how the classic hairy ball theorem blocks the existence of PIR in local domains (including free space), a special nonlocal metamaterial (MTM) in infinite and homogeneous nonlocal domain was shown to support a PIR pattern for arbitrarily oriented small dipoles. We proposed using this new fundamental existence theorem in order to construct a possible practical future PIR antenna. The proposed systems appear to lay beyond the capabilities of present day EM solvers since generic boundary conditions don't exist for nonlocal MTMs. We anticipate that future progress in this advanced and promising topic of nonlocal antennas will require collaboration between experimentalists and theorists.

\footnotetext{
${ }^{11}$ For a general theoretical discussion of these types of advanced nonlocal metamaterials, see [19].
} 


\section{REFERENCES}

[1] L. Felsen, Radiation and scattering of waves. Piscataway, NJ: IEEE Press, 1994.

[2] J. Schwinger et al., Classical electrodynamics. Reading, Mass: Perseus Books, 1998.

[3] T. Hansen and A. D. Yaghjian, Plane-Wave Theory of Time-Domain Fields: Near-Field Scanning applications. New York: IEEE Press, 1999.

[4] V. Guillemin and A. Pollack, Differential topology. Providence, R.I: AMS Chelsea Pub, 2010.

[5] S. Poslad, Ubiquitous computing: smart devices, environments and interactions. Chichester, U.K: Wiley, 2009.

[6] C. A. Balanis, Antenna Theory: Analysis and Design, 4th ed. Interscience: Wiley, 2015.

[7] K. Fujimoto and H. Morishita, Modern Small Antennas. Cambridge: Cambridge University Press, 2013.

[8] W. C. Jake, Ed., Microwave Mobile Communications (An IEEE Press Classic Reissue). Wiley-IEEE Press, 1994.

[9] B. P. Lathi and Z. Ding, Modern digital and analog communication systems. New York: Oxford University Press, 2019.

[10] V. Galindo and K. Green, "A near-isotropic circulary polarized antenna for space vehicles," IEEE Transactions on Antennas and Propagation, vol. 13, no. 6, pp. 872-877, 1965.

[11] J. Ahn, H. Jang, H. Moon, J.-W. Lee, and B. Lee, "Inductively Coupled Compact RFID Tag Antenna at $910 \mathrm{MHz}$ With Near-Isotropic Radar Cross-Section (RCS) Patterns," IEEE Antennas and Wireless Propagation Letters, vol. 6, pp. 518-520, 2007.

[12] Z. Su, K. Klionovski, R. M. Bilal, and A. Shamim, "3D printed nearisotropic asymmetric dipole antenna-on-package for IoT applications," in 2018 IEEE Indian Conference on Antennas and Propogation (InCAP), 2018, pp. 1-3.

[13] S. M. Radha, M. Jung, P. Park, and I.-J. Yoon, "Design of an Electrically Small, Planar Quasi-Isotropic Antenna for Enhancement of Wireless Link Reliability under NLOS Channels," Applied Sciences, vol. 10, no. $18,2020$.

[14] H. T. Ali, S. Amin, M. Amin, M. Maqsood, A. R. Maud, and M. Yusuf, "Design and Development of a Near Isotropic Printed Arc Antenna for Direction of Arrival (DoA) Applications," Electronics, vol. 10, no. 7, 2021.

[15] V. M. Agranovich, Y. R. Shen, R. H. Baughman, and A. A. Zakhidov, "Linear and nonlinear wave propagation in negative refraction metamaterials," Phys. Rev. B, vol. 69, p. 165112, April 2004.

[16] V. M. Agranovich, Y. N. Gartstein, and A. A. Zakhidov, "Negative refraction in gyrotropic media," Phys. Rev. B, vol. 73, p. 045114, Jan 2006.

[17] V. M. Agranovich and Y. Gartstein, "Spatial dispersion and negative refraction of light," Physics-Uspekhi, vol. 49, no. 10, p. 1029, 2006.

[18] S. Mikki and A. Kishk, "Nonlocal electromagnetic media: A paradigm for material engineering," in Passive Microwave Components and Antennas. InTech, April 2010.

[19] S. Mikki, "On the topological structure of nonlocal continuum field theories," Foundations, vol. 2, no. 1, pp. 20-84, 2022

[20] _ " "Theory of electromagnetic radiation in nonlocal metamaterials Part I: Foundations," Progress In Electromagnetics Research B, vol. 89, pp. 63-86, 2020.

[21] - "Theory of electromagnetic radiation in nonlocal metamaterials Part II: Applications," Progress In Electromagnetics Research B, vol. 89, pp. 87-109, 2020.

[22] S. Mikki, "Exact derivation of the radiation law of antennas embedded into generic nonlocal metamaterials: A momentum-space approach," in 2020 14th European Conference on Antennas and Propagation (EuCAP), 2020, pp. 1-5.

[23] S. Mikki, "Proca metamaterials, massive electromagnetism, and spatial dispersion," Annalen der Physik, vol. 533, no. 8, p. 2000625, June 2021.

[24] S. Mikki and Y. Antar, New Foundations for Applied Electromagnetics: The Spatial Structure of Fields. London: Artech House, 2016.

[25] V. Agranovich and V. Ginzburg, Crystal optics with spatial dispersion, and excitons. Berlin, Heidelberg: Springer Berlin HeidelbergImprint Springer, 1984.

[26] S. Mikki and Y. Antar, "A theory of antenna electromagnetic near fieldPart I," IEEE Transactions on Antennas and Propagation, vol. 59, no. 12 pp. 4691-4705, December 2011.

[27] C. H. Wilcox, "An expansion theorem for electromagnetic fields," Communications on Pure and Applied Mathematics, vol. 9, no. 2, pp. 115-134, May 1956.

[28] V. L. Ginzburg, The propagation of electromagnetic waves in plasmas. Oxford,New York: Pergamon Press, 1970.
[29] —, Theoretical physics and astrophysics. Oxford New York: Pergamon Press, 1979.

[30] S. Pekar, "The theory of electromagnetic waves in a crystal in which excitons are produced," JETP, vol. 6, no. 4, p. 785, May 1957.

[31] S. I. Pekar, Crystal optics and additional light waves. Menlo Park, Calif: Benjamin/Cummings Pub. Co, 1983.

[32] L. D. Landau and E. Lifshitz, Electrodynamics of continuous media. Oxford England: Butterworth-Heinemann, 1984.

[33] J. Jackson, Classical electrodynamics. New York: Wiley, 1999.

[34] S. Coleman, Quantum field theory: lectures of Sidney Coleman. New Jersey: World Scientific, 2019.

[35] L.-C. Tu, J. Luo, and G. T. Gillies, "The mass of the photon," Reports on Progress in Physics, vol. 68, no. 1, pp. 77-130, Nov 2005.

[36] v. J. Bladel, Electromagnetic fields. Wiley-Intersience, IEEE, 2007.

[37] S. Mikki and Y. Antar, "On the Fundamental Relationship Between the Transmitting and Receiving Modes of General Antenna Systems: A New Approach," IEEE Antennas and Wireless Propagation Letters, vol. 11, pp. 232-235, 2012.

[38] V. Ginzburg, "Electromagnetic waves in isotropic and crystalline media characterized by dielectric permittivity with spatial dispersion," JETP, vol. 7, no. 6, p. 1096, December 1958 